\title{
Efficacy of Potassium Bromide (As an Out-of-Date Drug) in Epilepsia Partialis Continua: A Brief Report
}

\author{
Reza Shervin Badv, ${ }^{1}$ Sara Memarian, ${ }^{2}$ Hossein Farshad Moghaddam, ${ }^{2}$ Behdad Gharib, ${ }^{2,}{ }^{*}$ Bahram \\ Yarali, ${ }^{2}$ Houman Alizadeh, ${ }^{2,3}$ and Morteza Heidari ${ }^{1,4}$ \\ ${ }^{1}$ Department of Pediatrics, Tehran University of Medical Sciences, Tehran, Iran \\ ${ }^{2}$ Children's Medical Center, Pediatrics Center of Excellence, Tehran, Iran \\ ${ }^{3}$ Department of Radiology, Tehran University of Medical Sciences, Tehran, Iran \\ ${ }^{4}$ Department of Pediatrics, Alborz University of Medical Sciences, Karaj, Iran \\ "Corresponding author: Behdad Gharib, Children's Medical Center, Pediatrics Center of Excellence, Tehran, Iran. Tel: +98-216147, Fax: +98-2166930024, E-mail: \\ behdad_gharib@yahoo.co.uk
}

Received 2016 April 08; Revised 2016 May 26; Accepted 2016 October 13.

Keywords: Refractory Seizure, Pachygyria, Epilepsia Partialis Continua, Potassium Bromide

\section{Dear Editor,}

Epilepsia partialis continua (EPC) is a term for focal motor clonic and/or myoclonic seizures, which last for days, months or longer (1).

Some of the reported causes of EPC are as follows: Cerebral tumors; lymphomas; metastasis; hemangiomas; multiple sclerosis; infectious mass lesions such as tuberculosis, vascular etiologies, vasculitis in lupus and in Sjogern syndrome; cortical dysplasias, specially hemimegalencephaly; tuberous sclerosis; linear sebaceous nevus syndrome; Sturge-Weber syndrome; widespread gliomatosis cerebri; paraneoplastic recurrent multiple encephalitis; cat scratch disease; non ketotic hyperglycemia; mitochondrial disorders; and hemiconvulsive hemiplegia epilepsy syndrome (HHE). Iatrogenic EPC can be induced by penicillin, azlocillin-cefotaxime and metrizamide. In addition to the conventional anti-epileptic drugs (AEDs), corticosteroids prescription may be indicated in EPC. Intravenous immunoglobulins, plasmapheresis and surgical intervention are other therapeutic modalities (2).

A six-year-old boy was brought to the pediatric neurology clinic of children's medical center because of increased frequency and intensity of the seizure activity.

He was born preterm from a twin pregnancy by cesarean delivery, his parents were not related, and the birth weight was 1700 grams. His parents did not recall the exact gestational age (they just remembered that he was born few weeks earlier than the estimated date), and the precise anthropometric measures at birth. He had a history of neonatal jaundice that lasted two weeks, and a vague record of blood sugar fluctuations during the first days of life. He had been admitted to hospitals many times for seizure attacks. He has been diagnosed as epileptic since he was two months old. The patient's linguistic ability was severely impaired.

He had been under observation in our neurologic clinic since a year ago when he had been hospitalized because of increased frequency of seizures, which presented by tonic clonic partial convulsion of the right upper and lower limbs, and right eye blinking for several hours a day. He was discharged with the diagnosis of symptomatic partial refractory epilepsy and the prescription of lamotrigine and primidone.

Brain magnetic resonance imaging was performed and revealed an asymmetry of cortical thickness between the right and left hemispheres, and a diffuse increased cortical thickness at the left hemisphere, which was more significant at the vertex. This finding was in favor of migrational abnormalities such as pachygyria (Figure 1). Mild ventriculomegaly and prominence of the right hemisphere sulci pointed to secondary atrophic changes from the longstanding seizure (Figure 2).

The patient had never been completely seizure free. He was discharged with right hemiparesis of the lower and upper limbs.

In the last admission to the hospital, he had seizures more than 10 times a day. The seizure pattern was tonicclonic convulsion of the right body side, accompanied with right lateral gaze.

He was admitted with the diagnosis of mixed type (myoclonic + partial) symptomatic epilepsy. The partial seizures continued and mostly occurred at night. He received many stat doses of phenobarbital, and phenytoin was also added to the medication list. As seizure frequency did not show the expected improvement, intravenous sodium valproate and midazolam continuous infusion were also administered. On the following days, en- 


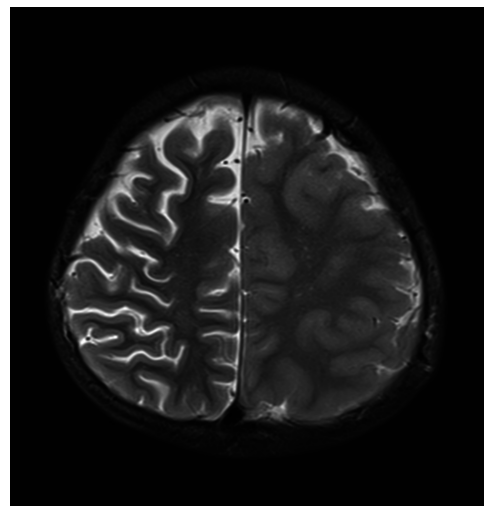

Figure 1. Brain Magnetic Resonance Imaging Revealed an Asymmetry of Cortical Thickness Between the Right and Left Hemispheres, a Diffuse Increased Cortical Thickness at the Left Hemisphere, Which Was More Significant at the Vertex in Favor of Migrational Abnormalities Such as Pachygyria

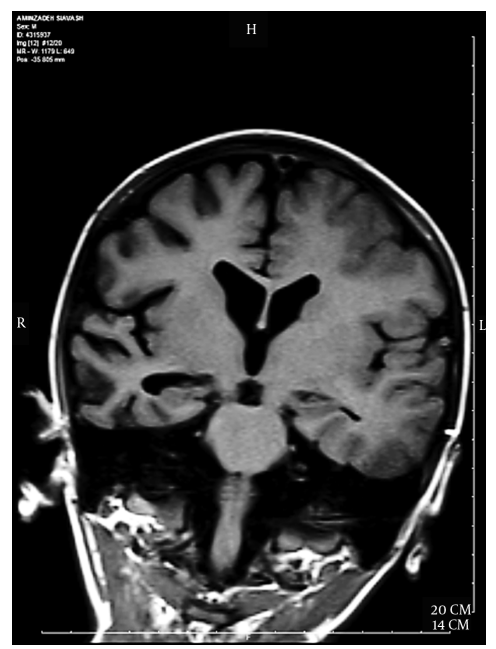

Figure 2. Mild Ventriculomegaly and Prominence of the Right Hemisphere Sulci Pointed to the Secondary Atrophic Changes From Longstanding Seizure

teral vigabatrin and pregabaline were also added and the doses gradually increased, and intravenous immunoglobulin was also prescribed.

Not observing any proper effect of the conventional AEDs, we prescribed potassium bromide $850 \mathrm{mg}$ per day, divided in two doses, and increased it to three doses of $850 \mathrm{mg}$ in the mornings, $425 \mathrm{mg}$ at evenings and $850 \mathrm{mg}$ at nights. By adding potassium bromide, the frequency of seizures reduced, and we started to reduce the dose of other medications gradually.

The patient was discharged on day 60 , with the following medications: Phenytoin tablets, sodium valproate syrup, phenobarbital tablets, and potassium bromide 850 $\mathrm{mg}$, one tablet in the mornings and half a tablet at nights.
Prior to the introduction of phenobarbital in 1912, bromide was the main anticonvulsant medication. Potassium bromide has a long half-life (12 - 14 days), and because reaching a steady blood level requires 40 - 50 days, it makes drug monitoring difficult. The toxic and therapeutic blood concentration levels are close to each other. The side effects of potassium bromide are drowsiness, low appetite, and skin rash. Moreover, in concentrations above $200 \mathrm{mg} / \mathrm{dL}$, adverse effects such as restlessness, headache, delirium and dementia, sometimes accompanied by hallucination, have been reported. A large intake of sodium chloride lowers the bromide level, but a low $\mathrm{NaCl}$ intake increases the bromide level, which could reach to toxic levels (3). The exact mechanism of potassium bromide effect has not been elucidated, but some studies proposed that this drug acts through gamma-aminobutyric acid (GABA) (4).

Our patient had EPC, which had been refractory to the conventional AEDs. The seizure started since early infancy. The brain anomaly (Pachygyria) was considered the main etiology for EPC. As the patient did not respond to the previous medications and routine antiepileptic drugs, we decided to try potassium bromide. Fortunately, the seizure frequency responded to potassium bromide. The result was a significant reduction in seizure frequency. In the follow-up visits, no increase was observed in seizure frequency one year after hospital discharge.

\section{References}

1. Mohamad A, Mikati A, Hani J. Seizures in Childhood. 20 ed. Philadelphia: Saunders; 2016. pp. 2823-56.

2. Epilepsy . A Comprehensive Textbook. 2 ed. Philadelphia: Lippincott Williams \& Wilkins; 2008.

3. Takayanagia M, Yamamotoa K, Nakagawaa H. Two successful cases of bromide therapy for refractory symptomatic localization-related epilepsy. Brain Dev. 2002;24(1):94-6. doi: 10.1016/S0387-7604(02)000232

4. Schmalbach B, Moeller B, von Spiczak S, Muhle H, Stephani U, Lang N. Seizure control in a patient with Dravet syndrome and cystic fibrosis. Epilepsy Behav Case Rep. 2013;1:42-4. doi: 10.1016/j.ebcr.2013.02.001. [PubMed: 25667824] 